\title{
Direct gating and mechanical integrity of Drosophila auditory transducers require TRPN1
}

Thomas Effertz ${ }^{1,3}$, Björn Nadrowski ${ }^{1,4}$, David Piepenbrock ${ }^{1}$, Jörg T. Albert², and Martin C. Göpfert ${ }^{1}$

1 Department of Cellular Neurobiology, University of Göttingen, Göttingen, Germany

2 UCL Ear Institute, London, London, UK

3 Present address: Department of Otolaryngology-Head and Neck Surgery, Stanford University, Stanford, USA.

4 Present address: Department of Theoretical Biological Physics, Saarland University, Saarbrücken, Germany

Correspondence should be addressed to J.T.A. (joerg.albert@ucl.ac.uk) or M.C.G (mgoepfe@gwdg.de).

The elusive transduction channels for hearing are directly gated mechanically by the pull of gating springs. We found that the transient receptor potential (TRP) channel TRPN1 (=NOMPC) is essential for this direct gating of Drosophila auditory transduction channels, and that the channel-spring complex is disrupted if TRPN1 is lost. This identifies TRPN1 as a mechanical constituent of the fly's auditory transduction complex that may act as channel and/or gating spring. 
Auditory transduction channels occur in series with gating springs that couple stimulus forces to their gates ${ }^{1,2}$. The hallmark of this force-activation is a nonlinear gating compliance that arises from the relaxation of the springs as the transduction channels gate ${ }^{1,2}$. Nonlinear gating compliances have been documented for vertebrate hair cells $s^{1,2}$ and the antennal ear of Drosophila ${ }^{3}$, but neither the underlying auditory transduction channels nor their gating springs have been identified yet $^{4}$. One candidate for an auditory transduction channel and its gating spring is TRPN1, which acts as a mechanotransduction channel in nematode touch receptors ${ }^{5}$ and bears an $\mathrm{N}$-terminal ankyrin spring ${ }^{6}$. TRPN1 has been implicated in the function of zebrafish auditory hair cells ${ }^{7}$ and the Drosophila ear ${ }^{8}$, but its importance for auditory transduction is uncertain: In hair cells, TRPN1 localizes to kinocilia that are dispensable for transduction ${ }^{9}$; and in the Drosophila ear insensitive sound-responses persist when TRPN1 is lost ${ }^{10}$. The latter sound-responses have been traced back to TRPN1-independent gravity/wind-sensitive cells in the ear of the fly that occur along with sensitive auditory sensory cells whose sensory function is abolished by the loss of TRPN1 ${ }^{10}$. To test whether the fly's TRPN1-dependent auditory sensory cells use TRPN1 for signal transduction or modulation we now analysed the nonlinear gating compliance in flies carrying mutations in nompC, which encodes TRPN $1{ }^{11}$.

To assess the gating compliance in the fly's auditory mechanics, we deflected its sound receiver formed by the distal part of the antenna ${ }^{3}$ with forces $F$ between -80 and $+80 \mathrm{pN}$ and measured its resulting displacement $X$ (ref. 3; Fig. 1a). In wild-type flies, this displacement characteristically peaks upon the onset of forcing before approaching a constant steady-state while forcing is maintained ${ }^{3}$ (Fig. 1a). The initial displacement peak reportedly reflects transducer gating, which nonlinearly reduces the antenna's stiffness, $K=\delta F / \delta X$, over that range of receiver displacements that 
make the channels gate ${ }^{3}$ (Fig. 1b). In the steady-state, this nonlinear gating compliance is no longer detectable, signalling that transducer adaptation is complete 3 (Fig. 1). We found that both the nonlinear gating compliance and the associated displacement peak are virtually lost in homozygous nomp $C^{2} \mathrm{cn}$ bw or nomp $C^{3} \mathrm{cn}$ bw null mutants but present in the genetic background strain, $c n$ bw, and in balanced nomp $C^{2} \mathrm{cn}$ bw/Cy cn and nompC $C^{3} \mathrm{cn}$ bw/Cy cn controls (Fig. 1).

Notwithstanding the loss of the gating compliance in nompC nulls, large antennal deflections evoked residual compound action potentials (CAPs) in their antennal nerves (Fig. 1). These residual CAPs were found to associate with a shallow gating compliance when we extended the forcing range to -550 to $+550 \mathrm{pN}$, resulting in antennal displacements between ca. -8 and $+8 \mu \mathrm{m}$ (Fig. 2a). Fitting this compliance with a symmetric gating spring model with two opposing transducer populations predicted an open probability for the transduction channels that, for probabilities exceeding the resting value of 0.5 , matched the amplitudes characteristics of the residual CAPs (Fig. 2a).

Over the extended displacement range, also wild-type (Supplementary Fig. 1) and control antennae (Fig. 2a) displayed a shallow gating compliance, in addition to their sharp gating compliance seen at small displacement amplitudes (Fig. 1b). This nonlinear behaviour and the associated CAPs could be reproduced when the symmetric gating spring model was supplemented with a second type of transducer, with both transducer types operating in parallel and differing in channel numbers and sensitivities to force (Supplementary Tab. 1). For $c n$ bw controls, fits of this 'twotransducer-type' model yielded $610 \pm 140$ sensitive and 94,000 $\pm 3,600$ insensitive transducers with single gating forces of $23 \pm 2 \mathrm{fN}$ and $2.2 \pm 0.7 \mathrm{fN}$, respectively (Supplementary Tab. 2). These figures compare to $48,000 \pm 24,000$ transducers with 
a single channel gating force of $2.4 \pm 0.7 \mathrm{fN}$ deduced with the 'one-transducer-type' model for nompC nulls. Hence, the fly's antenna reflects the direct gating of at least two types of transducers, and only insensitive transducer gating persists in nompC nulls.

The coexistence of two transducer types in the fly's hearing organ is paralleled by the coexistence of two types of sensory cells: ca. half of the 500 mechanosensory neurons of this organ mediate hearing whereas the others detect gravity and wind $^{12,13}$. Because only the former auditory neurons require TRPN1 for their mechanosensory function ${ }^{10}$, we reasoned that the sensitive TRPN1-dependent transducers are affiliated with these cells. To test this hypothesis, we selectively ablated the auditory neurons via ricin-toxin expression ${ }^{10,12}$ and found that only the shallow gating compliance persists (Fig. 2a). The 'one-transducer-type' model sufficed to describe this compliance (Supplementary Tab 1), yielding 62,200 $\pm 9,700$ insensitive transducers with a single channel gating force of $2.3 \pm 0.3 \mathrm{fN}$. Hence, ablating auditory neurons abolishes the gating of the sensitive transducers, identifying them as auditory transducers that reside in auditory sensory cells.

Loss of TRPN1 and auditory neurons impairs the gating of auditory transducers and, in addition, reduces the asymptotic stiffness, $K_{\infty}$, the antenna assumes at large displacements when the channels of each transducer population are all open or closed (Fig. 2a,b). According to the gating spring model, this asymptotic stiffness reflects the linear elasticity, $K_{\text {lin }}$, of the antennal joint and the neurons that proximally suspend the receiver and, in addition, the combined stiffness, $K_{G S}$, of the gating springs, $K_{\infty}=K_{\text {lin }}+K_{G S} . \quad K_{\infty}$ can be deduced from fits of the gating spring model and $K_{\text {lin }}$ can be inferred as the antenna's stiffness in steady-state when transducer adaptation seems complete. For $c n$ bw controls, we thus obtained $K_{\text {lin }}=52 \pm 2 \mu \mathrm{N} / \mathrm{m}$ 
and $K_{G S}=26 \pm 3 \mu \mathrm{N} / \mathrm{m}$ (Fig. 2b and Supplementary Tab. 2). In flies with ablated auditory neurons, $K_{\operatorname{lin}}(43 \pm 5 \mu \mathrm{N} / \mathrm{m})$ was significantly lower, signalling a loss of the ablated cells. $K_{G S}$ was $16 \pm 6 \mu \mathrm{N} / \mathrm{m}$, providing an estimate of the combined gating spring stiffness of the insensitive transducers and suggesting that the auditory transducers contribute some $10 \mu \mathrm{N} / \mathrm{m}$ to $K_{G S}$ in the controls (Fig. 2b). In nompC nulls, $K_{\text {lin }}(48 \pm 7 \mu \mathrm{N} / \mathrm{m})$ resembled that of $c n$ bw controls, documenting the mechanical integrity of neurons and joint. $K_{G S}(15 \pm 8 \mu \mathrm{N} / \mathrm{m})$, however, was significantly lowered by on average $11 \mu \mathrm{N} / \mathrm{m}$, which is close to the $10 \mu \mathrm{N} / \mathrm{m}$ assigned to the auditory transducers of the controls. This match, if non-coincidental, suggests that the loss of TRPN1 mechanically disrupts the auditory transducers or their connection with the antenna while leaving the ear's mechanics otherwise intact.

Additional evidence that TRPN1 is required for the mechanical integrity of auditory transducers was provided by nompC ${ }^{f 00642}$ hypomorphic mutants in which TRPN1 protein levels are reduced ${ }^{14}$ (Fig. 2b): judging from fits of the 'two channel type' model, only some 110 gating auditory transducers persist in these flies (Supplementary Tab. 2), and $K_{G S}(19 \pm 3 \mu \mathrm{N} / \mathrm{m})$ assumes values between those of controls and null mutants, indicating that some auditory transducers are disrupted mechanically while others remain intact. The inverse effect, that is a partial restoration of $K_{G S}$ and auditory transducer number, was observed in nomp $C^{3}$ null mutants when one copy of a UAS-nompC-L rescue construct ${ }^{15}$ was expressed in the ear's sensory cells (Fig. 2a,b). Expressing two copies of the construct fully restored $K_{G S}$ and the number of auditory transducers (Fig. 2a,b). Mean values of $K_{G S}$ correlated with auditory transducer numbers for different fly strains (Spearman's rank correlation coefficient $\rho=0.94$, two-tailed significance level $\alpha=0.05$ ), and plotting them against each other revealed a linear relation between both (Fig. 2c). According 
to this relation, each auditory transducer contributes some $16 \mathrm{nN} / \mathrm{m}$ to the receiver's stiffness, and without auditory transducers $K_{G S}$ drops to $17 \mu \mathrm{N} / \mathrm{m}$, which is close to the respective figures obtained for flies with ablated auditory neurons and for nompC nulls. Hence, in these latter flies $K_{G S}$ can be attributed solely to the insensitive transducers, documenting that the auditory transducers are abolished or decoupled from the receiver if the auditory neurons or TRPN1 are lost. Neurons and receiver are properly connected in nompC nulls as witnessed by the linear elasticity of their receivers (Fig. 2b). This narrows down the site of the mechanical disruption to the transducers and associated force-transmitting structures within the auditory sensory cells.

Ever since TRPN1 was implicated in the mechanosensory function of Drosophila tactile bristles ${ }^{11}$, it has been hypothesized to be one of the elusive transduction channels for hearing $4,7,8,10$. TRPN1 was found to be required for auditory sensory cell function in zebrafish ${ }^{7}$ and Drosophila ${ }^{8,10}$, but neither this TRP nor any other channel protein has been demonstrated to be required for auditory transduction channel function ${ }^{4,10}$. Such requirement is established here for Drosophila TRPN1, identifying it as the first ion channel protein for auditory transduction. According to our results, TRPN1 forms the fly's auditory transduction channels or couples forces to them, acting as -or in series with- their gating springs. Both functions seem plausible given that TRPN1 can act as mechanotransduction channel ${ }^{5}$ and bears an ankyrin spring ${ }^{6}$.

The coexistence of TRPN1-dependent and -independent transduction channels in the fly's auditory organ shows that one ear can harbour distinct transducer types and explains why this organ partially retains its mechanosensitivity when TRPN1 is lost ${ }^{8,10}$ : lack of TRPN1 eliminates auditory sensory cell function by disrupting auditory transducers, whereas TRPN1-independent transduction in the ear's gravity/wind- 
sensitive cells persists. Mammals lack TRPN1 and, accordingly, must use other channel proteins for mechanotransduction. TRPN1-independent mechanotransduction channels also occur in the Drosophila ear and the present study sets the stage for genetically dissecting their in vivo function.

\section{Online Methods}

\section{Fly strains.}

The following strains were used: Oregon $\mathrm{R}$ (wild-type), nomp $C^{2} \mathrm{cn}$ bw and nomp $C^{3}$ cn bw (nompC nulls), cn bw (genetic background controls), nomp $C^{2} \mathrm{cn} b w / C y c n$ and nomp $C^{3}$ cn bw/Cy cn (balanced controls), JO2;eyFLP/+;JO15/UFWTRA19 (auditory neurons ablated, 'AN>ricin'); $\mathrm{PBac}\{\mathrm{WH}\}$ nomp $C^{\text {f00642 }}$ (hypomorphic mutants); nompC $C^{3}$, UAS-NompC-L/nompC 3 ;NP0761/+ and nompC ${ }^{3}$, UAS-NompC-L;NP0761/+ (partial and full rescue ('nompC- ,1 $\times$ N>nompC-L' and 'nompC- ,2 $\times N>n o m p C-L$ '), respectively). Flies were tested 4 to 10 days upon hatching and maintained according to German Federal regulations (license Gen.Az 501.40611/0166/501).

\section{Data acquisition.}

Displacements of the the fly's antenna were imposed using electrostatic force ${ }^{3,16}$. To allow for both positive and negative forcing, the electrical potential of the fly was lifted to ca. $100 \mathrm{~V}$ against ground via a tungsten charging electrode in the thorax ${ }^{3,16}$. The resulting antennal displacement, $\boldsymbol{X}$, was measured in non-loading condition at the tip of the antenna's arista using a Polytec (Waldbronn, Germany) PSV-400 scanning 
laser Doppler vibrometer with a DD-5000 displacement decoder ${ }^{3,16}$. Antennal nerve responses were recorded via a tungsten electrode inserted between antenna and head $^{3,16}$. Flies from different strains were tested in a randomized order. Signals were sampled at a rate of $100 \mathrm{kHz}$ for offline analysis.

\section{Data analysis.}

To correct for stimulus-uncorrelated artefacts as caused by muscular movements, we first extracted the time traces of the receiver's displacement response to identical step stimuli and calculated the average displacements and corresponding standard deviations. To detect outliers, we used the Grubbs test ${ }^{17}$, which assumes a normal distribution of the data and rejects outliers based on this assumption. The Grubbs test was applied iteratively until no more outliers were detected. The statistic $G$ of the Grubbs test is defined as

$$
\boldsymbol{G}=\frac{\max _{i=1, \ldots, N}\left\lfloor X_{i}-\bar{X}\right]}{S},
$$

where $\boldsymbol{X}_{\boldsymbol{i}}$ is the displacement amplitude for response $\boldsymbol{i}$ at time $\boldsymbol{t}$ relative to the step onset, $\boldsymbol{N}$ is the number of samples, and $\bar{X}$ and $S$ are the corresponding average displacement amplitude and standard deviation, respectively. The hypothesis of no outliers was rejected at the significance level of $\boldsymbol{\alpha}$ (in our case, $\boldsymbol{\alpha}=0.5$ ) if

$$
G>\frac{N-1}{\sqrt{N}} \sqrt{\frac{t^{2} \alpha /(2 N), N-2}{N-2+t^{2} \alpha /(2 N), N-2}},
$$


where $t^{2}{ }_{\alpha /(2 N), N-2}$ denotes the upper critical value of the t-distribution with $\boldsymbol{N}-\mathbf{2}$ degrees of freedom and a significance level of $\boldsymbol{\alpha} /(2 \mathrm{~N})$.

After having rejected outliers, time traces of the average displacement and nerve responses to force steps of different amplitudes were separately plotted. The external force experienced by the receiver was deduced from its maximum acceleration immediately upon the onset of forcing, $\ddot{X}_{\text {onset }}$, and its apparent mass, $\boldsymbol{m}$ (ca. $\mathbf{5}$. $\mathbf{1 0}^{-9} \boldsymbol{g}$, refs. 3,18): $\boldsymbol{F}=\boldsymbol{m} \cdot \ddot{\boldsymbol{X}}_{\text {onset }}=\mathbf{5} \cdot \mathbf{1 0}^{-\mathbf{9}} \boldsymbol{g} \cdot \ddot{\boldsymbol{X}}_{\text {onset }}$. The steady state displacement, $\boldsymbol{X}_{\text {steady }}$, that the receiver assumed during prolonged forcing was deduced as the asymptotic value of an exponential fit to the measured displacement response (ref. S9). The corresponding steady-state stiffness, $\boldsymbol{K}_{\text {steady }}$, was calculated as $\boldsymbol{K}_{\text {steady }}=$ $\boldsymbol{\partial}\left(\boldsymbol{m} \ddot{\boldsymbol{X}}_{\text {onset }}\right) / \boldsymbol{\partial} \boldsymbol{X}_{\text {steady. }}$. The dynamic stiffness of the receiver during the initial displacement peak, $\boldsymbol{X}_{\text {peak }}$, was correspondingly calculated as $\boldsymbol{K}_{\text {peak }}=\boldsymbol{\partial}\left(\boldsymbol{m} \ddot{\boldsymbol{X}}_{\text {onset }}-\right.$ $\left.\boldsymbol{m} \ddot{\boldsymbol{X}}_{\text {peak }}\right) / \boldsymbol{\partial} \boldsymbol{X}_{\text {peak }}$, whereby the force experienced by the receiver, $\boldsymbol{m} \ddot{\boldsymbol{X}}_{\text {onset }}$, was corrected for inertial effects, $\boldsymbol{m} \ddot{\boldsymbol{X}}_{\text {peak }}$, caused by the receiver's mass ${ }^{3}$.

The apparent mass of individual antennal receivers reportedly varies between ca. $\mathbf{2} \cdot \mathbf{1 0}^{-12}$ and $\mathbf{7} \cdot \mathbf{1 0}^{-12} \mathrm{~kg}$, with an average of ca. $\mathbf{5} \cdot \mathbf{1 0}^{-12} \mathrm{~kg}$ (ref. 18). Fixing the mass to the latter value shifts all the variability that arises from individual mass differences to stiffness, hampering the identification of differences in receiver stiffness between fly strains. To compensate for this inter-individual variability, we adjusted the mass of each individual antennal receiver so that its steady-state stiffness $\boldsymbol{K}_{\text {steady }}$ matched the average value obtained for the respective strain. In line with this adjustment, the masses of individual receivers had to be altered by maximally $15 \%$, which is well within the range of the reported inter-individual mass 
variation (ca. $40 \%$, ref. 18). To compare receiver mechanics between different strains, their steady-state stiffness, $\boldsymbol{K}_{\text {steady, }}$ and dynamic stiffness at the displacement peak, $\boldsymbol{K}_{\text {peak }}$. were plotted against the stimulus force (Figs. 1,2).

\section{Modeling}

The fly's antennal sound receiver is proximally suspended by an antennal joint and two opposing populations of mechanosensory sensilla. Following previous studies $^{1,3,16}$, we describe this system with a symmetric gating spring model with two opposing populations of transducer complexes and a parallel spring of stiffness $K_{\text {lin }}$. This latter, linear stiffness represents the combined linear elasticity of mechanosensilla and the antennal joint. Each transducer population is assumed to consist of $\mathrm{N} / 2$ transducer complexes modules that are arranged in parallel, with each complex consisting of one transduction channel that occurs in series with one gating spring of stiffness $k$. The combined stiffness that the gating springs contribute to the receiver's mechanics, $K_{G S}$, can be written as $K_{G S}=N \gamma^{2} k$, where $\gamma$ is the projection factor that relates antennal mechanics and molecular events ${ }^{3,16}$. The open probability of each channel population is defined as $p_{o}(X)=1 /\left(1+e^{-z\left(X-X_{0}\right) / k_{B} T}\right)$, where $z$ is the change in force in a single gating spring as the channel opens, $X_{0}$ the antennal displacement at which the open probability is one-half, $k_{B}$ is the Boltzmann constant, and $T$ is the absolute temperature. Because $X_{0} \cong 0$ for all receivers that were examined in this study, the open probability can be written as $p_{o}(X)=1 /(1+$ $\left.e^{-z X / k_{B} T}\right)$. Being arranged perpendicularly, the open probabilities of the opposing transducer populations, $p_{o}(X)$ and $p_{o}(-X)=1-p_{o}(X)$, are inversed: when the channels of one population are mostly in the open state, those of the opposing 
population will mostly be closed. After correcting for inertial effects due to the receiver's mass (see above), the force-displacement relation of this symmetric system can be written as

$$
F=K_{\infty} X-N z p_{o}(X)+\frac{N}{2} z,
$$

yielding a displacement-dependent receiver stiffness $K(X)$ of

$$
K(X)=K_{\infty}-\left(\frac{N z^{2}}{k_{B} T}\right) p_{o}(X)\left(1-p_{o}(X)\right) .
$$

In this 'one-transducer-type' model $K_{\infty}$ represent the asymptotic stiffness the receiver assumes at large forcing amplitudes when the channels of one transducer population are all open and those of the opposing population are all closed. The negative term on the right hand side of the equation describes the gating compliance, which renders the receiver's stiffness minimal when the open probabilities of both transducer populations are $0.5 . \quad K_{\infty}$ is determined the linear elasticity, $K_{\text {lin }}$, of the antennal joint and the neurons that suspend the receiver and the combined stiffness, $K_{G S}$, of the gating springs, $K_{\infty}=K_{l i n}+K_{G S}$. We directly deduced $K_{\text {lin }}$ from the steady-state response of the receiver, because this response was linear and because the steady-state stiffness equals $K_{\text {lin }}$ if transducer adaptation is complete ${ }^{1,3}$. $K_{G S}$, provides a measure of the mechanical integrity of the transduction modules, and was deduced as $K_{\infty}-K_{\text {lin }}$. Note that loss of transducer gating will linearize the stiffness of the receiver, $K(X)=K_{\infty}$, and that mechanically disrupting the transducer complex will render $K_{G S}$ zero, so that $K(X)=K_{\text {lin }}$. 
Our analysis suggests that the fly's ear houses at least two different types that differ in their sensitivities to force. The respective channels are likely to be arranged in parallel and their open probabilities are $p_{o_{S}}(X)$ and $p_{o_{S}}(-X)$ and $p_{o_{i}}(X)$ and $p_{o_{i}}(-X)$, respectively, where the subscripts $s$ and $i$ indicate sensitive and insensitive. Supplementing equation 4 with a second transducer type leads to

$$
K(X)=K_{\infty}-\left(\frac{N_{s} z_{s}^{2}}{k_{B} T}\right) \cdot p_{o_{s}}\left(1-p_{o_{S}}\right)-\left(\frac{N_{i} z_{i}^{2}}{k_{B} T}\right) \cdot p_{o_{i}}\left(1-p_{o_{i}}\right) .
$$

In this 'two-transducer-type' model, $\mathrm{N}_{\mathrm{s}}$ and $\mathrm{N}_{\mathrm{i}}$ are the channel numbers of the sensitive and insensitive transducer types, respectively, $p_{o_{s}}$ and $p_{o_{i}}$ are the corresponding open probabilities, and $\mathrm{z}_{\mathrm{s}}$ and $\mathrm{z}_{\mathrm{i}}$ are the corresponding changes in force in a single gating spring as the associated channel opens. All channels are assumed to gate independently of one another, and $K_{\infty}$ equals $K_{G S}+K_{\text {lin }}$, with $K_{G S}$ representing the combined gating spring stiffness of the sensitive and insensitive transducer channels, $K_{G S}=\mathrm{K}_{\mathrm{GS}_{\mathrm{S}}}+\mathrm{K}_{\mathrm{GS}_{\mathrm{i}}}$. Average channel open probabilities can be calculated as

$$
\left(\mathrm{p}_{\mathrm{o}_{\mathrm{S}}}+\mathrm{p}_{\mathrm{o}_{\mathrm{i}}}\right) / 2=1 /\left(\frac{1}{\left(1+\mathrm{e}^{\left(-\frac{\mathrm{z}_{\mathrm{S}} \mathrm{X}}{k_{B} T}\right)}\right)}+\frac{1}{\left(1+\mathrm{e}^{\left(-\frac{\mathrm{z}_{\mathrm{i}} \mathrm{X}}{k_{B} T}\right)}\right)}\right) / 2
$$

and, for the respective opposing populations, as $1-\left(\left(\mathrm{p}_{\mathrm{o}_{\mathrm{s}}}+\mathrm{p}_{\mathrm{o}_{\mathrm{i}}}\right) / 2\right)$. Loss of one of the two transducer types will re-simplify Eq. 5 to Eq. 4, abolishing the gating compliance associated with the gating of the respective channels and reducing the receiver's stiffness by the combined stiffness of the respective gating springs. 


\section{Model selection.}

To test whether the compliance of the antennal receiver in a given fly strain is better described by the 'one channel type' (Eq. 4) or the 'two channel type' (Eq. 5) model, we fitted the pooled dynamic stiffnesses obtained for this strain with both models and determined the respective sum of the squared residuals, Rss,

$$
R s s=\sum_{i=0}^{n}\left(y_{i}-f_{i}\right)^{2},
$$

where $y$ is the measured and $f$ the simulated value.

To assess the goodness of the fits, we used the Akaike information criterion with correction for finite sample size (AICc; ref. 19),

$$
A I C c=A I C+\frac{2 k(k+1)}{n-k-1} \text { with } A I C=n \ln \left(\frac{R s s}{n}\right)+2 k .
$$

Eq. 8

Here, $n$ is the number of data points and $k$ is the number of free parameters. Based on the differences $\Delta=A I C c_{r}-A I C c_{\min }$ obtained for the $r=2$ models, their respective Akaike weigths, $w_{i}$, were calculated as

$$
w_{i}=\frac{\exp \left(-\Delta_{i} / 2\right)}{\sum_{r=1}^{2} \exp \left(-\Delta_{i} / 2\right)} .
$$

Akaike weights provide a measure of the probability that the data is better approximated by one model than by the other and can assume figures between 0 
(low probability) and 1 (high probability). Values of $A I C c$ and $w_{i}$ obtained for the different fly strains are provided in Supplementary Tab 1.

\section{Statistics.}

One-tailed Mann Whitney U-Tests with Bonferroni correction for multiple comparisons were used for statistical analysis. Difference was considered statistically significant when $\mathrm{P}<0.05$. The data are represented as mean \pm s.d.

1. Howard, J.\& Hudspeth, A.J. Neuron 1, 189-199 (1988).

2. Tinevez, J.Y., Jülicher, F. \& Martin, P. Biophys J 93, 4053-4067 (2007).

3. Albert, J.T., Nadrowski, B. \& Göpfert, M.C. Curr. Biol. 17, 1000-1006 (2007).

4. Arnadottir, J. \& Chalfie, M. Annu. Rev. Biophys. 39, 111-137 (2010).

5. Kang L. et. al. Neuron 67, 381-391 (2010).

6. Howard, J. \& Bechstedt, S. Curr. Biol. 14, R224-R226 (2004).

7. Sidi, S., Friedrich, R.W. \& Nicolson, T. Science 301, 96-99 (2003).

8. Göpfert, M.C., Albert, J.T., Nadrowski, B. \& Kamikouchi, A. Nat. Neurosci. 9, 999-1000 (2006).

9. Shin J.B. et al. Proc. Natl. Acad. Sci. USA 102, 12572-12577 (2005).

10. Effertz, T., Wiek, R. \& Göpfert, M.C. Curr. Biol. 21, 592-597 (2011).

11. Walker, R.G., Willingham, A.T. \& Zuker, C.S. Science 287, 2229-2234 (2000).

12. Kamikouchi, A. et al. Nature 458,165-171 (2009).

13. Yorozu, S. et al. Nature 458, 201-205 (2009).

14. Lee, J., Moon, S., Cha, Y. \& Chung, Y.D. PLoS ONE 5, e11012 (2010). 
15. Cheng, L.E. et al. Neuron 67, 373-380 (2010).

16. Nadrowski, B., Albert, J.T. \& Göpfert, M.C. Curr. Biol. 18, 1365-1372 (2008).

17. Grubbs, F.E. Technometrics 11, 1-21 (1969).

18. Göpfert, M.C., Humphris, A.D., Albert, J.T., Robert, D. \& Hendrich, O. Proc. Natl. Acad. Sci. USA 102, 325-330 (2005).

19. Burnham, K.P. \& Anderson, D.R. Model selection and multimodel interference: a practical information-theoretic approach $2^{\text {nd }}$ edn. (Springer, New York, 2002).

\section{Author contributions}

T.E. performed the experiments with the help of D.P., B.N. devised models, and T.E. analyzed the data. J.T.A. devised methods and performed pilot studies. M.C.G. supervised the project and wrote the manuscript with T.E., B.N., D.P., and J.T.A.

\section{Acknowledgements}

We thank Richard Walker, Li Cheng, Yuh-Nung Jan, the Bloomington Stock Centre, the Exelixis Collection at Harvard Medical School, and the Nippon Consortium for fly strains. This work was supported by the Volkswagen Foundation (B.N.), the German Academic Foundation (D.P.), BBSRC (BB/G004455/1, J.T.A), and the German Science Foundation (SFB889-A1, to M.C.G.).

\section{Competing interests statement:}

The authors declare no competing financial interests. 


\section{Figure Legends}

Figure 1. Correlates of transducer gating depend on TRPN1. (a) Force-induced displacements of the antennal sound receiver (top) and CAPs (middle). Arrows: peak (red) and steady-state (blue) displacement of the antenna and CAP amplitude (brown). Blue line: Exponential fit to deduce the steady state displacement. (b) Antennal stiffness (top) and relative CAP amplitudes (bottom) as functions of the antennal displacement. Symbols: dynamic stiffness at the displacement peak (red), steady-state stiffness (blue), and CAP amplitudes (brown). Relative CAP voltage (V) amplitudes are calculated as $\left(\boldsymbol{V}-\boldsymbol{V}_{\min }\right) /\left(\boldsymbol{V}_{\max }-\boldsymbol{V}_{\text {min }}\right)$. For sample sizes $(\geq 4$ flies per strain), see Supplementary Tab. 2.

Figure 2. TRPN1 is required for auditory transducer gating and integrity. (a) Stiffness of the antennal sound receiver (top) and associated CAPs (bottom) over an extended range of antennal displacements. Colour coding as in Fig. 1. Solid lines: fitted gating spring models (top) and predicted open probabilities ( $\boldsymbol{p}_{\boldsymbol{o}}$, bottom). Hatched lines: asymptotic stiffness $\left(\boldsymbol{K}_{\infty}\right)$ and linear elasticity $\left(\boldsymbol{K}_{\text {lin }}\right)$ of the receiver. (b) $\boldsymbol{K}_{\infty}, \boldsymbol{K}_{\text {lin }}$, and gating spring stiffness $\boldsymbol{K}_{G S}$ in different fly strains (means \pm 1 s.d.). Hatched lines: values of the genetic background strain; red arrows: significant deviations thereof $(p<0.05$, Mann-Whitney U-tests (one-tailed) with Bonferroni correction). (c) $\boldsymbol{K}_{G S}$ as function of the number of auditory transducers in different fly strains (means \pm 1 s.d.; sample size $\geq 4$ flies per strain). Red line: linear regression (slope $=16 \mathrm{nN} / \mathrm{m}, \mathrm{Y}$-Intercept $\left.=17 \mu \mathrm{N} / \mathrm{m} ; \mathrm{R}^{2}=0.9\right)$. Arrows: $\boldsymbol{K}_{G S}$ in nompC nulls and flies with ablated auditory neurons $(A N>r i c i n)$, which both are excluded from the analysis. For sample sizes and fit parameter values, see Supplementary Tab. 2. 


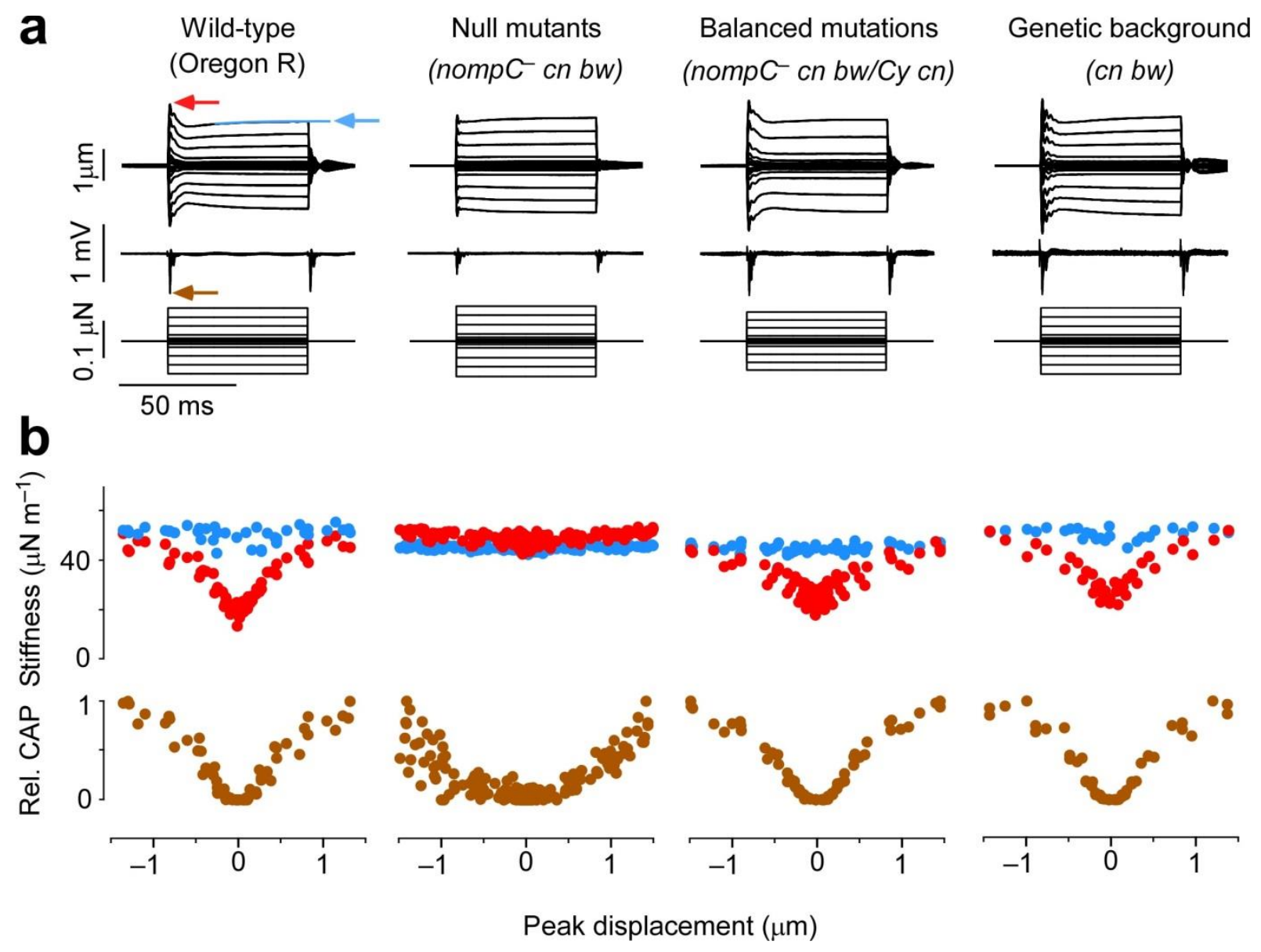

Fig. 1 

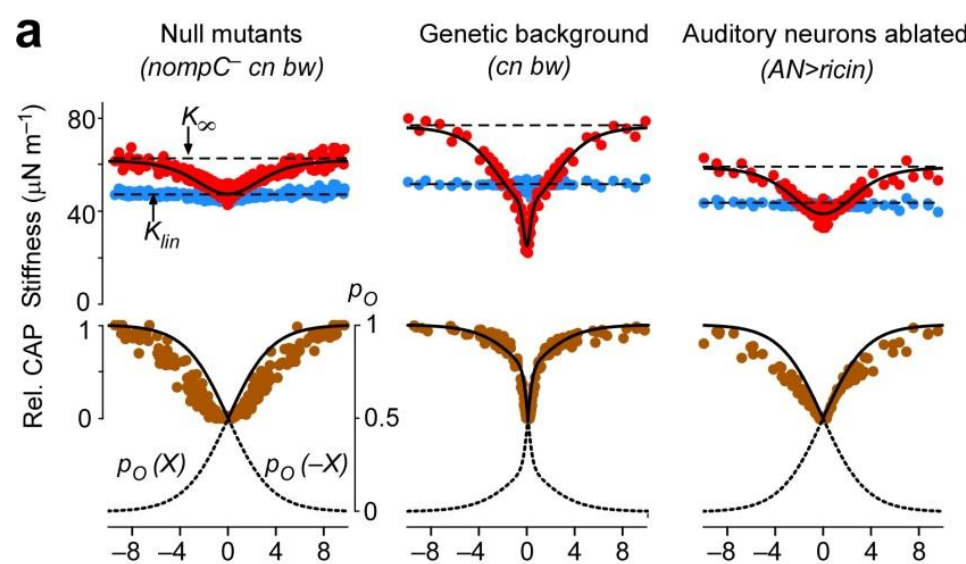

b (AN>ricin)
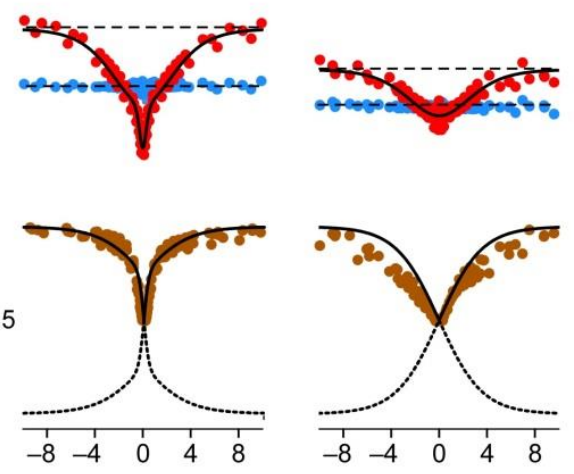

Peak displacement $(\mu \mathrm{m})$

Hypomorphic mutants

Partial rescue

Rescue

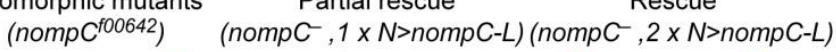

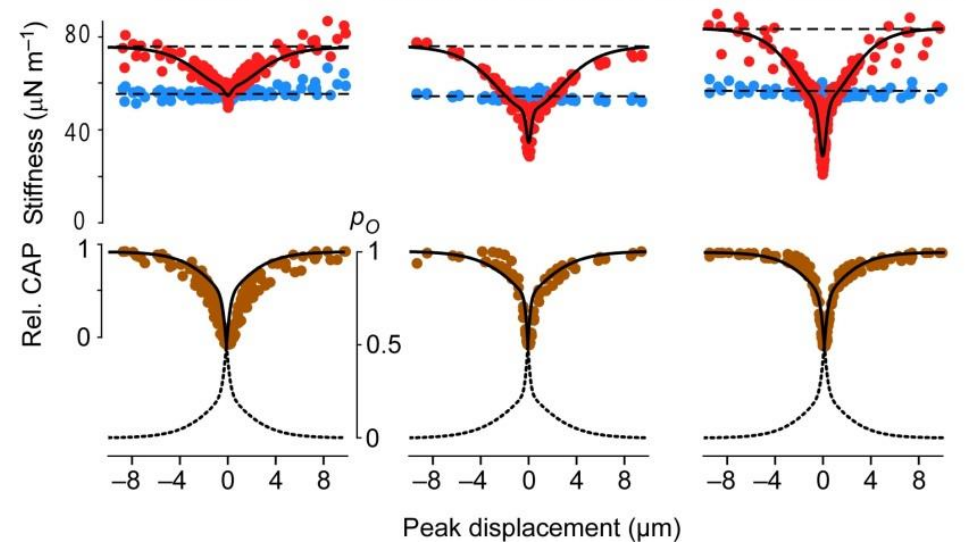

C

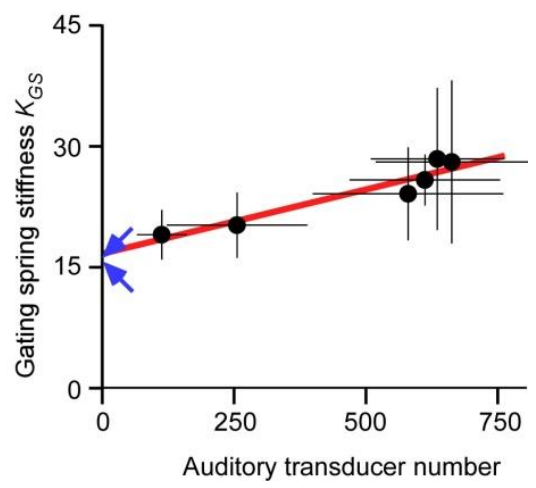

Fig.2 


\section{Supplementary Information}

\section{Direct gating and mechanical integrity of Drosophila auditory transducers require TRPN1}

Effertz, T., Nadrowski, B., Piepenbrock, D., Albert, J.T., and Göpfert, M.C.
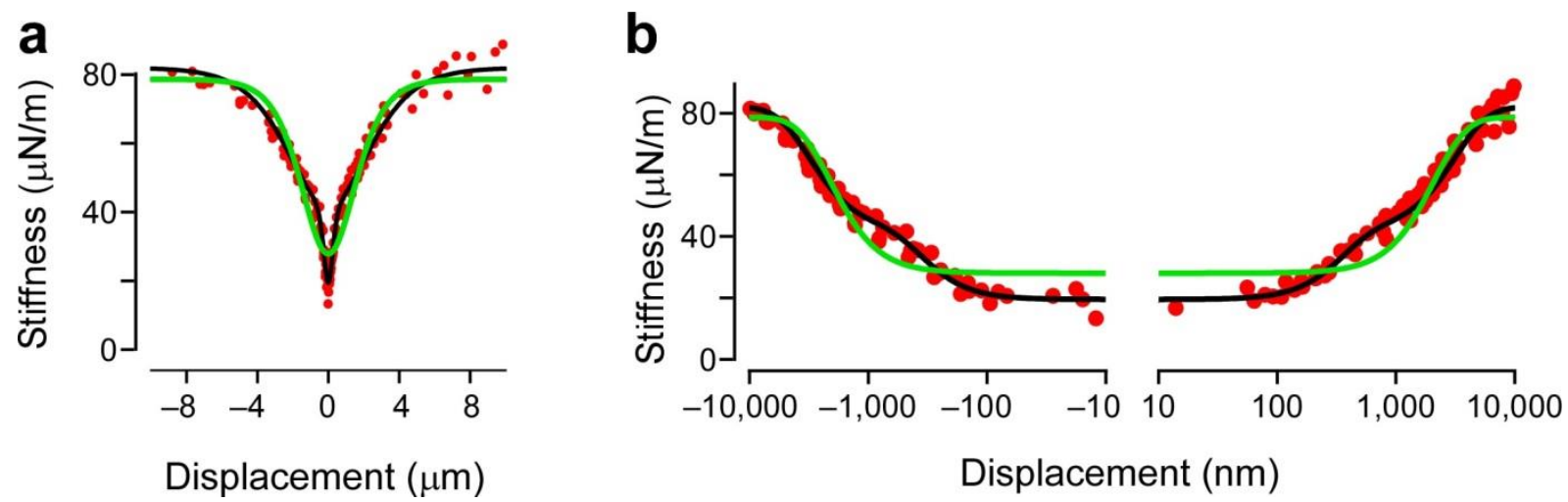

Supplementary Figure 1. Displacement-dependent dynamic stiffness of Oregon $\mathbf{R}$ wild-type receivers fitted with 'one-transducer-type' (green line) and 'twotransducer-type' (black line) models. (a) lin-lin plot. (b) lin-log plot of the same data to highlight deviations between data and fits at small receiver displacements. Sample size: 5 animals.

\begin{tabular}{|l|c|ccccc|}
\cline { 2 - 6 } \multicolumn{1}{c|}{} & \multicolumn{3}{c}{ AIC c } & \multicolumn{2}{c|}{$\boldsymbol{W}_{\boldsymbol{i}}$} \\
\hline wild-type & $\boldsymbol{n}$ & $k=3$ & $k=5$ & $k=3$ & $k=5$ \\
null mutants & 135 & -1401 & -1576 & 0.0 & 1.0 \\
genetic background & 549 & -6935 & -6905 & 1.0 & 0.0 \\
balanced mutations & 108 & -1144 & -1273 & 0.0 & 1.0 \\
auditory neurons ablated & 160 & -1724 & -1818 & 0.0 & 1.0 \\
gain-of-function mutants & 135 & -1583 & -1579 & 0.9 & 0.1 \\
partial rescue & 225 & -2696 & -2737 & 0.0 & 1.0 \\
full rescue & 108 & -1152 & -1221 & 0.0 & 1.0 \\
\hline
\end{tabular}

Supplementary Table 1. Model selection. Akaike information criterion with correction for finite sample size $(A / C c)$ and corresponding Akaike weights $\left(W_{i}\right)$ obtained by fitting the dynamic stiffnesses of the antennal receivers of different fly strains with gating spring models with one and two different channel types. $n$ is the number of data points and $k$ the number of model parameters. Akaike weights of the better one of the two models are highlighted in red. For each strain, the better model was used to produce the fits in Figure 2. Respective parameter values are presented in Supplementary Tab. 2. 


\begin{tabular}{|c|c|c|c|c|c|c|c|c|}
\hline & $N_{s}$ & $\begin{array}{c}z_{s} \\
(\mathrm{fN}) \\
\end{array}$ & $\overline{N_{i}}$ & $\begin{array}{c}z_{i} \\
(\mathrm{fN})\end{array}$ & $\begin{array}{c}\boldsymbol{K}_{\infty} \\
(\mu \mathrm{N} / \mathrm{m})\end{array}$ & $\begin{array}{c}\boldsymbol{K}_{\text {lin }} \\
(\mu \mathrm{N} / \mathrm{m})\end{array}$ & $\begin{array}{c}\boldsymbol{K}_{\boldsymbol{G} \boldsymbol{S}} \\
(\mu \mathrm{N} / \mathrm{m})\end{array}$ & $N_{\text {flies }}$ \\
\hline Wild-type & $660 \pm 140$ & $24 \pm 2$ & $\begin{array}{c}91,400 \pm \\
7,600\end{array}$ & $2.7 \pm 0.1$ & $80 \pm 3$ & $52 \pm 10$ & $28 \pm 10$ & 5 \\
\hline null mutants & - & - & $\begin{array}{l}48,000 \pm \\
24,000\end{array}$ & $2.4 \pm 0.7$ & $63 \pm 2$ & $48 \pm 7$ & $15 \pm 8$ & 23 \\
\hline $\begin{array}{l}\text { genetic } \\
\text { background }\end{array}$ & $610 \pm 140$ & $23 \pm 2$ & $\begin{array}{c}94,000 \pm \\
3,600\end{array}$ & $2.2 \pm 0.7$ & $77 \pm 2$ & $52 \pm 2$ & $26 \pm 3$ & 4 \\
\hline balanced mutations & $580 \pm 281$ & $20 \pm 3$ & $\begin{array}{c}95,300 \pm \\
12,900\end{array}$ & $2.2 \pm 0.2$ & $71 \pm 2$ & $47 \pm 6$ & $24 \pm 6$ & 6 \\
\hline $\begin{array}{l}\text { auditory neurons } \\
\text { ablated }\end{array}$ & - & - & $\begin{array}{c}62,200 \pm \\
9,700\end{array}$ & $2,3 \pm 0.3$ & $59 \pm 3$ & $43 \pm 5$ & $16 \pm 6$ & 5 \\
\hline $\begin{array}{l}\text { gain-of-function } \\
\text { mutants }\end{array}$ & $110 \pm 50$ & $21 \pm 1$ & $\begin{array}{c}55,800 \pm \\
25,900\end{array}$ & $2.5 \pm 0.7$ & $75 \pm 2$ & $56 \pm 2$ & $19 \pm 3$ & 8 \\
\hline partial rescue & $260 \pm 130$ & $28 \pm 3$ & $\begin{array}{l}101,400 \pm \\
9700\end{array}$ & $2.1 \pm 0.1$ & $75 \pm 2$ & $54 \pm 3$ & $20 \pm 4$ & 4 \\
\hline full rescue & $640 \pm 130$ & $28 \pm 2$ & $\begin{array}{c}52,800 \pm \\
18,000\end{array}$ & $3.3 \pm 0.6$ & $86 \pm 12$ & $57 \pm 8$ & $28 \pm 14$ & 4 \\
\hline
\end{tabular}

Supplementary Table 2. Parameter values (means \pm 1 s.d.) deduced by fitting receiver mechanics with gating spring models. For parameters, genotypes, and modeling, see Supplementary Methods online. For model selection, see Supplementary Tab. 1. $\boldsymbol{N}_{\text {flies }}$ refers to the sample size (number of examined antennae, whereby only one antenna was tested per fly). 